\title{
Adipose tissue dysfunction and alcoholic liver disease
}

\begin{abstract}
Alcoholic liver disease (ALD) remains an important health problem in the United States. The disease process is featured by early steatosis, steatohepatitis (steatosis with inflammatory cells infiltration and necrosis), with some individuals ultimately progressing to fibrosis/cirrhosis. Although the disease progression is well characterized, no FDA-approved therapies are currently available to halt or reverse this process in humans. The mechanisms underlying the initiation and progression of ALD are complex and multifactorial. Emerging evidence supports that adipose tissue dysfunction contributes to the pathogenesis of ALD. It has been long known that aberrant hepatic methionine metabolism is a major metabolic abnormality induced by chronic alcohol exposure and plays an etiological role in the pathogenesis of ALD. The recent studies in our group documented the similar effect of chronic alcohol drinking in adipose tissue. In this article, we briefly reviewed the recent research progress in the field with a focus on how abnormal methionine metabolism in adipose tissue contributes to adipose tissue dysfunction and liver damage.
\end{abstract}

Keywords: alcohol, liver, adipose, methionine, lipolysis, methylation
Volume I Issue | - 2015

\section{Zhenyuan Song}

Department of Kinesiology and Nutrition, University of Illinois,

USA

Correspondence: Zhenyuan Song, Department of Kinesiology and Nutrition, Department of Pathology, University of Illinois,

Chicago, USA, Email song2008@uic.edu

Received: January 20, 20I5 | Published: February 28, 2015
Abbreviation: ALD, alcoholic liver disease; SAM, s-adenosyl methionine; SAH, s-adenosyl homocyteine; HSL, hormone-sensitive lipase; PP2A, protein phosphates 2A; SREBP, sterol regulatory element binding proteins; AMPK, amp-activated protein kinase; PPAR, peroxisome proliferator-activated receptor

\section{Alcoholic Liver Disease (ALD)}

Although much progress has been made over last three decades of research on the mechanisms underlying alcoholic liver disease (ALD), it remains an important health problem in the United States. ALD ranks among the major causes of morbidity and mortality in the world, and affects millions of patients worldwide each year. ${ }^{1}$ The disease process is characterized by early steatosis, steatohepatitis (steatosis with inflammatory cells infiltration and necrosis), with some individuals ultimately progressing to fibrosis/cirrhosis. Although the disease progression is well characterized, there is currently no FDAapproved therapy available to halt or reverse this process in humans.

\section{Pathogenesis of Early-Stage ALD}

Hepatic steatosis, characterized by excess fat accumulation in hepatocytes, is the most common and earliest response of the liver to chronic alcohol consumption. Although "pure" steatosis is clinically considered to be a benign condition, excessive fat accumulation makes hepatocytes vulnerable to the attack of "the second hit," such as pro inflammatory cytokines and oxidative stress, leading to the progression to steatohepatitis.,3 The mechanisms involved in the development of alcohol-induced hepatic steatosis are multifactorial. Sterol regulatory element binding proteins (SREBP)-1c, a master transcription factor controlling de novo lipogenesis, is up regulated in the liver of mice chronically exposed to ethanol-containing diet. ${ }^{4}$ Importantly, liver-specific knockout of SREBP-1c protect mice against alcohol-induced fatty liver and liver damage, ${ }^{5}$ supporting the notion that enhanced hepatic de novo lipogenic process plays a pivotal role in alcohol-triggered fat accumulation in the liver. Moreover, chronic alcohol exposure is associated with impaired fatty acid $\beta$-oxidation in hepatocytes, contributing to fat accumulation in hepatocytes. Suppressions of both adenosine monophosphate-activated protein kinase (AMPK) and peroxisome proliferator-activated receptor-alpha (PPAR-alpha), two regulatory proteins of fatty acids oxidation, are mechanistically involved in this process. ${ }^{6,7}$ Furthermore, long-term alcohol consumption is reported to enhance uptakes of free fatty acids and triglyceride-rich lipoproteins by hepatocytes ${ }^{8,9}$ and impair hepatic very-low-density lipoprotein (VLDL) secretion, ${ }^{10}$ thereby contributing to fatty liver after chronic alcohol exposure.

\section{Adipose Tissue Dysfunction and ALD}

Although it has been well-established that chronic alcohol consumption exerts a detrimental effect on hepatic fat synthesis and disposal, leading to the development of hepatic steatosis, emerging evidence supports that adipose tissue dysfunction also plays an important role in the pathogenesis of ALD. In the clinic setting, it has been reported that visceral fat accumulation is positively related to the onset of alcoholic liver damage and body mass index (BMI) represents an independent risk factor for fibrosis in alcoholic patients. ${ }^{11-14}$ Moreover, adipose tissue inflammation is correlated with the severity of pathological features in the liver of patients with ALD. ${ }^{13}$ Experimentally, long-term alcohol consumption is associated with adipose tissue oxidative stress, insulin resistance, inflammation, adipocyte cell death, and adiponectin decline. ${ }^{15-19}$ Chronic alcohol feeding results in hyper-lipolysis (degradation of triglycerides) in adipose tissue, leading to elevated circulation free fatty acids concentrations and a significant loss of white adipose tissue. ${ }^{8}$ A recent study demonstrated that moderate obesity and alcohol synergistically induced steatohepatitis, ${ }^{20}$ further supporting the critical role of adipose tissue (dys) function in the development of ALD. Importantly, both rosiglitazone (a PPAR-gamma agonist mainly targeting adipocytes) $)^{21}$ and recombinant adiponectin (an adipokine exclusively secreted by adipocytes ${ }^{22}$ improved ALD, suggesting that improving adipose tissue function represents a potential therapeutic approach for ALD. 


\section{Aberrant \\ methionine \\ metabolism contributes to adipose tissue hyper-lipolysis in ALD}

\section{Methionine metabolism abnormality in ALD}

Aberrant hepatic methionine metabolism is a major metabolic abnormality induced by chronic alcohol exposure and plays an etiological role in the pathogenesis of ALD. ${ }^{23-26}$ As illustrated in Figure 1, intracellular methionine metabolism involves two major pathways, transmethylation reaction and transsulfuration reaction. The first step in methionine metabolism is the formation of S-adenosyl methionine (SAM) in a reaction catalyzed by methionine adenosyl-transferase (MAT). Under physiological conditions, most of the SAM generated per day is used in transmethylation reactions in which methyl groups are added to a vast number of molecules, including DNA, RNA, phospholipids, histones, and other proteins, via specific methyltransferases. In this process, SAM is converted to S-adenosylhomocysteine (SAH), followed by homocyteine (Hcy) and cysteine, a precursor for glutathione biosynthesis, via transsulfuration pathway. SAH is a potent competitive inhibitor of most methyltransferases studied and decreased SAM: SAH ratio has been widely employed as an indicator of suppressed transmethylation reactions. ${ }^{27,28}$ While chronic alcohol exposure leads to hepatic SAM deficiency, both SAH and HCY are increased in the liver in response to alcohol.

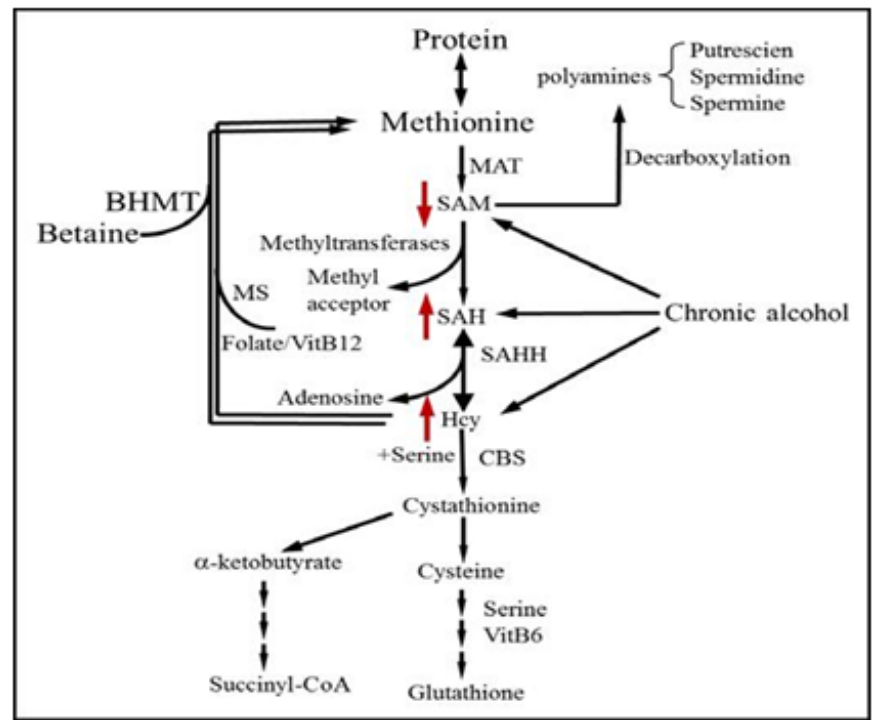

Figure I Intracellular methionine metabolism: Chronic alcohol consumption causes SAM deficiency, but enhancement of homocysteine and SAH.

Abbrevations: MAT, methionine adenosyl-transferase; SAM, s-adenosyl methionine; SAH, s-adenosylhomocysteine; Hcy, homocysteine; CBS, cystathionine beta synthase; $\mathrm{SAHH}$, s-adenosylhomocysteine hydrolase; MS, methionine synthase; $\mathrm{BHMT}$, betaine-homocysteine methyltransferase.

\section{Aberrant adipose methionine metabolism and hyper- lipolysis in adipose tissue}

Cellular/molecular mechanisms underlying alcohol-triggered adipocyte dysfunction are not completely known. Increased oxidative stress due to CYP2E1 over expression in adipose tissue represented one of the mechanisms. ${ }^{19}$ Studies using both genetic and dietary animal models provided evidence supporting that hyper homocysteinemia (HHcy) is associated with adipose tissue dysfunction, ${ }^{29-32}$ suggesting that methionine metabolism regulates adipose tissue function. We are the first to report that, similar to its effect on the liver, chronic alcohol feeding induces methionine metabolism abnormality in adipose tissue, which is characterized by SAM deficiency, and Hcy and SAH accumulation, leading to significant decrease of SAM/ SAH ratio, a strong indicator of inhibitory transmethylation reactions (hypomethylation). We subsequently showed that Hcy accumulation in adipose tissue contributes to adiponectin decline in ALD via a mechanism involving ER stress induction ${ }^{16}$ and adipogenesis suppression via inhibiting PPAR-gamma transactivation. ${ }^{33}$ Furthermore, we provided evidence supporting that intracellular hypomethylation status in adipocytes in the setting of chronic alcohol feeding contributes to adipose tissue hyper-lipolytic response in ALD via suppressing protein phosphatase $2 \mathrm{~A}(\mathrm{PP} 2 \mathrm{~A})$ activity, leading to hormone sensitive lipase over activation (Figure 2). ${ }^{34}$ Our data support that rectification of methionine metabolism through dietary supplementation of betaine protects against alcohol-induced liver damage, at least partially via improving adipose tissue function. Taken together, the recent research in our group suggests that aberrant methionine metabolism in adipocytes contributes to alcohol-elicited adipose tissue dysfunction and liver damage.

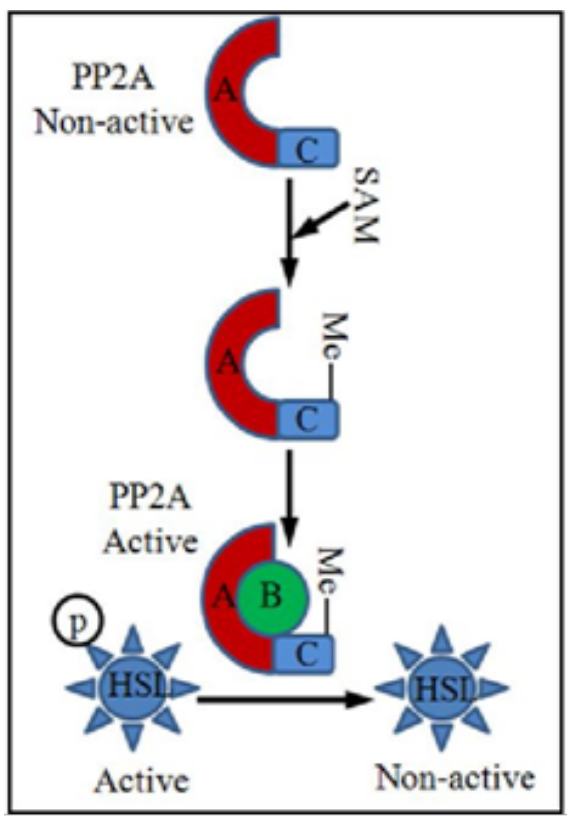

Figure 2 SAM-dependent methylation reactions are required for PP2A activation, which dephosphorylates (inhibits) HSL. Chronic alcohol consumption induces intracellular hypomethylation status in adipocytes, which suppresses PP2A activity, leading to uncontrolled HSL activation.

SAM, s-adenosylmethionine; Me, methyl group; PP2A, protein phosphatase 2a; HSL, hormone sensitive lipase.

\section{Conclusion}

Emerging evidence shows that adipose tissue plays an important role in both initiation and progression of liver damage induced by chronic alcohol consumption. The recent studies in our laboratory provide initial evidence suggesting that methionine metabolism abnormality contributes to alcohol-associated adipose tissue malfunction. Although much progress has been made, the exact underlying cellular/molecular mechanisms involved in adipose tissue dysfunction in ALD remain to be elucidated. Drugs targeting both adipose tissue and the liver represent ideal choices for the treatment of ALD. 


\section{Acknowledgements}

None.

\section{Conflict of interest}

Author declares that there is no conflict of interest.

\section{References}

1. Grant BF, Dufour MC, Harford TC. Epidemiology of alcoholic liver disease. Semin Liver Dis. 1988;8(1):12-25.

2. Stewart S, Jones D, Day CP. Alcoholic liver disease: new insights into mechanisms and preventative strategies. Trends $\mathrm{Mol} \mathrm{Med}$. 2001;7(9):408-413.

3. Reddy JK, Rao MS. Lipid metabolism and liver inflammation. II. Fatty liver disease and fatty acid oxidation. Am J Physiol Gastrointest Liver Physiol. 2006;290(5):852-858

4. You M, Fischer M, Deeg MA, et al. Ethanol induces fatty acid synthesis pathways by activation of sterol regulatory element-binding protein (SREBP). J Biol Chem. 2002;277(32):29342-29347.

5. Ji C, Chan C, Kaplowitz N. Predominant role of sterol response element binding proteins (SREBP) lipogenic pathways in hepatic steatosis in the murine intragastric ethanol feeding model. J Hepatol. 2006;45(5):717-724.

6. You M, Matsumoto M, Pacold CM, et al. The role of AMP-activated protein kinase in the action of ethanol in the liver. Gastroenterology. 2004;127(6):1798-1808

7. Fischer M, You M, Matsumoto M, et al. Peroxisome proliferator-activated receptor alpha (PPARalpha) agonist treatment reverses PPARalpha dysfunction and abnormalities in hepatic lipid metabolism in ethanol-fed mice. J Biol Chem. 2003;278(30):27997-28004 .

8. Zhong W, Zhao Y, Tang Y, et al. Chronic alcohol exposure stimulates adipose tissue lipolysis in mice: role of reverse triglyceride transport in the pathogenesis of alcoholic steatosis. Am J Pathol. 2012;180(3):998-1007.

9. Wang Z, Dou X, Li S, et al. Nuclear factor (erythroid-derived 2)-like 2 activation-induced hepatic very-low-density lipoprotein receptor overexpression in response to oxidative stress contributes to alcoholic liver disease in mice. Hepatology. 2014;59(4):1381-1392.

10. Sugimoto T, Yamashita S, Ishigami M, et al. Decreased microsomal triglyceride transfer protein activity contributes to initiation of alcoholic liver steatosis in rats. J Hepatol. 2002;36(2):157-162.

11. Hart CL, Morrison DS, Batty GD, et al. Effect of body mass index and alcohol consumption on liver disease: analysis of data from two prospective cohort studies. BMJ. 2010;340:c1240.

12. Tsai J, Ford ES, Zhao G, et al. Cooccurrence of obesity and patterns of alcohol use associated with elevated serum hepatic enzymes in US adults. J Behav Med. 2011;35(2):200-210.

13. Loomba R, Bettencourt R, Barrett-Connor E. Synergistic association between alcohol intake and body mass index with serum alanine and aspartate aminotransferase levels in older adults: the Rancho Bernardo Study. Aliment Pharmacol Ther. 2009;30(11-12):1137-1149.

14. Shen Z, LiY, Yu C, et al. A cohort study of the effect of alcohol consumption and obesity on serum liver enzyme levels. Eur J Gastroenterol Hepatol. 2010;22(7):820-825

15. Sebastian BM, Roychowdhury S, Tang H, et al. Identification of a cytochrome P4502E1/Bid/C1q-dependent axis mediating inflammation in adipose tissue after chronic ethanol feeding to mice. J Biol Chem. 2011;286(41):35989-35997.
16. Song Z, Zhou Z, Deaciuc I, et al. Inhibition of adiponectin production by homocysteine: a potential mechanism for alcoholic liver disease. Hepatology. 2008;47(3):867-879.

17. Kang L, Chen X, Sebastian BM, et al. Chronic ethanol and triglyceride turnover in white adipose tissue in rats: inhibition of the anti-lipolytic action of insulin after chronic ethanol contributes to increased triglyceride degradation. J Biol Chem. 2007;282(39):28465-28473.

18. Chen X, Sebastian BM, Nagy LE. Chronic ethanol feeding to rats decreases adiponectin secretion by subcutaneous adipocytes. $\mathrm{Am} \mathrm{J}$ Physiol Endocrinol Metab. 2007;292(2):E621-628.

19. Tang H, Sebastian BM, Axhemi A, et al. Ethanol-induced oxidative stress via the CYP2E1 pathway disrupts adiponectin secretion from adipocytes. Alcohol Clin Exp Res. 2012;36(2):214-222.

20. Xu J, Lai KK, Verlinsky A, et al. Synergistic steatohepatitis by moderate obesity and alcohol in mice despite increased adiponectin and p-AMPK. J Hepatol. 2011;55(3):673-682.

21. Sun X, Tang Y, Tan X, et al. Activation of peroxisome proliferatoractivated receptor- $\gamma$ by rosiglitazone improves lipid homeostasis at the adipose tissue-liver axis in ethanol-fed mice. Am J Physiol Gastrointest Liver Physiol. 2012;302(5):G548-557.

22. Xu A, Wang Y, Keshaw H, et al. The fat-derived hormone adiponectin alleviates alcoholic and nonalcoholic fatty liver diseases in mice. $J$ Clin Invest. 2003;112(1):91-100.

23. Barak AJ, Beckenhauer HC, Kharbanda KK, et al. Chronic ethanol consumption increases homocysteine accumulation in hepatocytes. Alcohol. 2001;25(2):77-81.

24. Mato JM, Camara J, Fernandez de Paz J, et al. S-adenosylmethionine in alcoholic liver cirrhosis: a randomized, placebo-controlled, double-blind, multicenter clinical trial. J Hepatol. 1999;30(6):1081-1089.

25. Lu SC, Huang ZZ, Yang $H$, et al. Changes in methionine adenosyltransferase and S-adenosylmethionine homeostasis in alcoholic rat liver. Am JPhysiol Gastrointest Liver Physiol. 2000;279(1):G178-185.

26. Song Z, Zhou Z, Uriarte S, et al. S-adenosylhomocysteine sensitizes to TNF-alpha hepatotoxicity in mice and liver cells: a possible etiological factor in alcoholic liver disease. Hepatology. 2004;40(4):989-997.

27. Mato JM, Alvarez L, Ortiz P, et al. S-adenosylmethionine synthesis: Molecular mechanisms and clinical implications. Pharmacol Ther. 1997;73(3):265-280.

28. Chiang PK, Gordon RK, Tal J, et al. S-Adenosylmethionine and methylation. FASEB J. 1996;10(4):471-480.

29. Gupta S, Kruger WD. Cystathionine beta-synthase deficiency causes fat loss in mice. PLoS One. 2011;6(11):e27598.

30. Mikael LG, Wang XL, Wu Q, et al. Hyperhomocysteinemia is associated with hypertriglyceridemia in mice with methylenetetrahydrofolate reductase deficiency. Mol Genet Metab. 2009;98(1-2):187-194.

31. Li Y, Zhang H, Jiang C, et al. Hyperhomocysteinemia promotes insulin resistance by inducing endoplasmic reticulum stress in adipose tissue. $J$ Biol Chem. 2013;288(14):9583-9592.

32. Li Y, Jiang C, Xu G, et al. Homocysteine upregulates resistin production from adipocytes in vivo and in vitro. Diabetes. 2008;57(4):817-827.

33. Wang Z, Dou X, Yao T, et al. Homocysteine inhibits adipogenesis in 3T3L1 preadipocytes. Exp Biol Med (Maywood). 2011;236(12):1379-1388.

34. Dou X, Xia Y, Chen J, et al. Rectification of impaired adipose tissue methylation status and lipolytic response contributes to hepatoprotective effect of betaine in a mouse model of alcoholic liver disease. $\mathrm{Br} J$ Pharmacol. 2014;171(17):4073-4086. 